\title{
Graphic File Formats for Web Virtual Globe
}

\author{
Sergey Presnyakov ${ }^{1}$, Grigory Boyarshinov ${ }^{1}$, Tatyana Borovskaya ${ }^{1}$ and Alena Rybkina ${ }^{1,2}$ \\ ${ }^{1}$ The Geophysical Center of the Russian Academy of Sciences, 3 Molodezhnaya Str., Moscow, 119296, Russia \\ ${ }^{2}$ The Schmidt Institute of Physics of the Earth of the Russian Academy of Sciences, 10-1 Bolshaya Gruzinskaya \\ Str., Moscow, 123995, Russia
}

\begin{abstract}
In this paper various graphic formats were reviewed as resources for web virtual globe. Interactive spherical visualization (ISV) platform called "Orbus" was considered as an example of a graphic resources repository for web virtual globe. Various raster and vector static and dynamic cartographic layers can be set as resources of applications of this type. Such data should fulfill requirements for high informativeness with a minimum disk volume. In this paper traditional formats used for geodata storing, as well as becoming popular formats that can be adapted to work with geodata were explored. As a result of the analysis of the characteristics of common raster formats, the optimized raster format WebP (in the lossless specification), as well as the associated WebM media container, were selected as the most efficient. As a result of analysis of the characteristics of common vector formats, conclusions about their limitations in visual design of the virtual globe were made.
\end{abstract}

\section{Keywords}

Virtual globe, graphic format, raster graphics, vector graphics.

\section{Introduction}

The Geophysical Center of the Russian Academy of Sciences (GC RAS) is successfully developing ISV web platform [1], which inherits the main features from hardware-software complex (HSC) "Orbus" [2] and provides access to the spherical slides for an expanding list of software clients: "OrbusTouch" spherical projection screen, "OrbusWeb" virtual globe, "OrbusAR" augmented reality applications.

Main purpose of such applications is the study of planetary processes and phenomena, as they look from space. These data are available for use in an interactive form from a variety of devices without the specifics of the GIS domain. Data as real or model representation can be stored via raster images (relief, infrastructure, etc.), or vector cartographic layers (Figure 1).

Since the main feature of the ISV web platform is global processes presentation, it is important to work with the small scale of planets and store layers in raster format in high resolution, made in a certain projection, or in vector format.

"OrbusWeb" and "OrbusAR" virtual globe clients are browser-based, so their development depends on different browser requirements for the processing of different formats of graphic resources.

GraphiCon 2021: 31st International Conference on Computer Graphics and Vision, September 27-30, 2021, Nizhny Novgorod, Russia EMAIL: s.presnyakov@gcras.ru (S. Presnyakov); g.boyarshinov@gcras.ru (G. Boyarshinov); t.borovskaya@gcras.ru (T. Borovskaya); a.rybkina@gcras.ru (A. Rybkina)

ORCID: 0000-0001-8710-5494 (S. Presnyakov); 0000-0003-0062-4350 (G. Boyarshinov); 0000-0003-4892-204X (T. Borovskaya); 0000-0002-9223-5936 (A. Rybkina)

(c) (i) (C) 2021 Copyright for this paper by its authors.

Use permitted under Creative Commons License Attribution 4.0 International (CC BY 4.0).

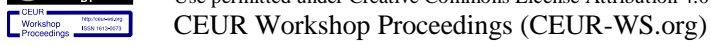




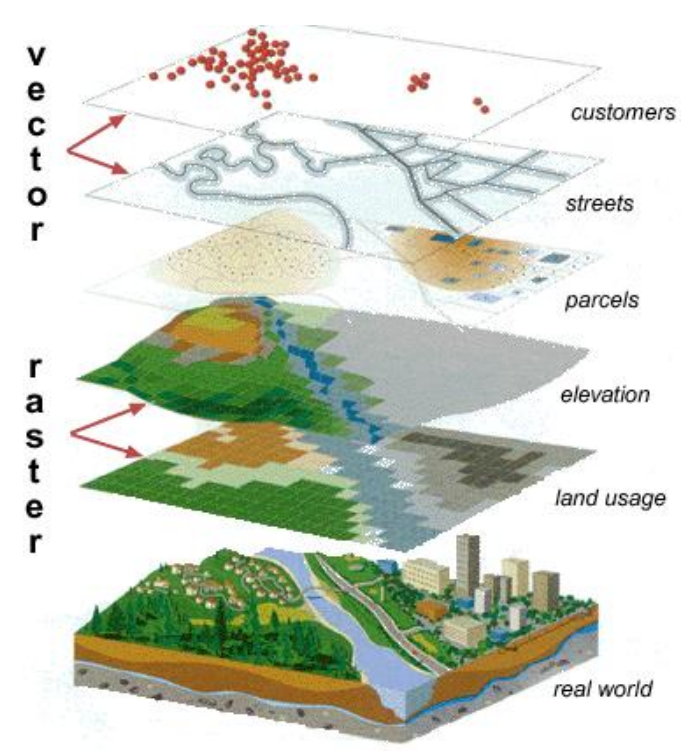

Figure 1: Raster and vector cartographic data layers [3]

\section{Initial formats of graphic resources}

HSC "Orbus" set the trend for the development of the ISV web platform. The software part of the Complex is a Desktop application associated with a spherical projection screen, which supports a number of graphic data formats as described below.

\subsection{Raster}

Compositions based on remote sensing are often raster data sources in GIS. For raster format it is convenient to transfer data in the form of an array, where each pixel corresponds to a specific coordinate. The image is most often built in a cylindrical equidistant projection (Figure 2). The color of the pixel can be used to encode the desired value (heat map), for example, the pressure distribution on the Earth's surface.

3-D Model

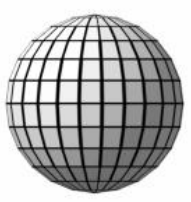

$p=(x, y, z)$

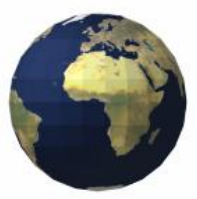

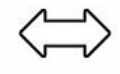

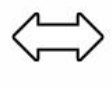

UV Map

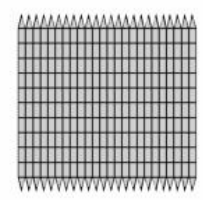

$p=(u, v)$

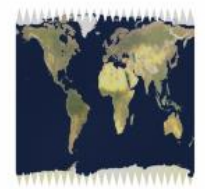

Texture

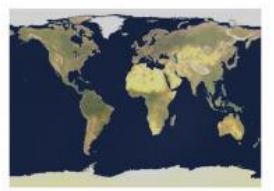

Figure 2: An example of overlaying a two-dimensional texture (coordinates $U, V$ ) on a threedimensional globe (coordinates $X, Y, Z$ ) [4]

One of the main features of raster format is the data compression mechanism that allows to reduce the file size. These mechanisms can be separated into two categories: lossy compression and lossless compression. Lossless compression ensures that all information is preserved, but not always effectively reduces file size. For example, for images close to white noise, the resulting size exceeds the original size. In lossy compression, some of the original information is lost, so the image after compression is not identical to the original. 
The choice of raster formats was made according to the support of data source formats, among various Internet services, such as NASA storage [5, 6, 7], taking into account formats traditionally used by GC RAS. In addition, for HSC "Orbus" DDS format were tested - a specialized format for storing textures. The following is a brief description of the raster formats used for the functioning of HSC "Orbus".

\subsubsection{PNG}

PNG (Portable Network Graphics) - free format supported by all platforms for storing bitmaps. The latest version of the standard was recommended by the World Wide Web Consortium in 2003 [8].

PNG implements lossless compression, which is important when working with geodata, where each pixel contains certain information. it outperforms JPEG and GIF in color quality, but file size is usually large.

PNG can work with the following color schemes:

- $\quad$ color indexing (up to 256 colors can be encoded);

- $\quad$ grayscale (up to 16 bits per raster);

- $\quad$ full color RGB image (three 16-bit channels);

- multilevel transparency (up to 16 bits).

PNG supports EXIF and XMP metadata, which allows you to add your own tags with the necessary information to the file.

Of the limitations, PNG does not support working with layers (the alpha channel can be limitly used for this) and animation.

\subsubsection{JPEG}

JPEG was created by Joint Photographic Experts Group in 1992. Currently, the JPEG format is supported by ISO, the latest version of the standard was developed in 2019 [9]. The first specification of 1992 is freely available [10].

JPEG was specially designed for storing photographic images and uses lossy compression and is effective at storing low-contrast images.

JPEG does not work with transparency, colors are saved in RGB format, with a depth of 8 bits for each channel.

EXIF, XMP metadata can be attached to JPEG files. Layers and animations are not supported.

\subsubsection{GeoTIFF and TIFF}

GeoTIFF (Geographic Tag Image File Format) is an extension of TIFF 6.0 format by means of geotags describing the type of projection, coordinate systems, etc. The Open Geospatial Consortium published GeoTIFF version 1.1 in 2019 [11].

TIFF 6.0 was designed in 1992 [12], the specification is currently owned by Adobe Inc. There are extended format options that are not supported by all programs.

TIFF is a format that contains an image and its description with tags. It supports lossless compression; the certain compression algorithm can be selected by the user. In advanced versions, TIFF supports lossy compression, PNG and JPEG method compression.

It works with indexed palette, grayscale, RGB, CMYK, etc., transparency is supported. Color depth can be set up to 16 bits per channel, but there are extensions that increase this range. Due to such wide capabilities, TIFF can be used whenever color control is important.

There is an extension that allows TIFF to store multiple layers (or bands) of an image in a single file [13]. Animation directly in TIFF is not supported, but such multi-page files can be recreated using specialized software as animation.

\subsubsection{GIF}


GIF (Graphics Interchange Format) was developed for transmission of raster images over networks, the last version of the standard was adopted in 1989 [14]. GIF uses a lossless compression algorithm.

When working with color, an indexed palette of 256 colors is used. In this case, the color in the palette can be declared transparent.

The preservation of this format to this day is largely due to its support for animation.

Metadata can be added using XMP technology.

\subsubsection{DDS}

Initially, DDS (DirectDraw Surface) was developed by Microsoft for use in DirectX, currently is ia also supported in OpenGL [15], however not on all platforms of its WebGL subset [16].

DDS can be stored uncompressed or lossy compressed. In this case, the files are saved in a form in which they can be directly sent to the video card. For other formats, you need to recover the compressed file first and then convert it, so DDS is processed faster by a video card. The color depth can be selected up to 32 bits per channel when saved uncompressed. When compressing, the depth is selected up to 8 bits per pixel.

DDS does not support animation; meta information is not attached to this file type.

\subsubsection{MP4/H.264}

An animated planetary surface is most often represented as an ordered sequence of bitmap images from the formats described above. An alternative option is a video stream using.

MP4 (MPEG-4 Part 14) is a media container format that is part of the MPEG-4 standard. The first version of the format, ISO/IEC 14496-1:2001, was released in 2001, the final version, ISO/IEC 1449614:2003, in 2003 [17].

Like most modern media containers, MP4 provides the ability to display video over the Internet. MP4 files can contain standard metadata and also supports XMP metadata format. The container allows to package multiple video streams.

MP4 container supports the following data compression formats:

- $\quad$ MPEG-I Part 3 (H.266/VVC);

- $\quad$ MPEG-H Part 2 (H.265/HEVC);

- $\quad$ MPEG-4 Part 10 (H.264/AVC);

- $\quad$ MPEG-4 Part 2;

- H.262/MPEG-2 Part 2;

- H.261/MPEG-1 Part 2.

Currently, the most widely used codec is MPEG-4 Part 10, which is used in digital high-definition television HDTV and in many other areas of digital video. The popularity, in particular, is explained by the fact that in 2010 the developers announced the free use of this codec [18]. It should be noted that the H.264 video stream has a number of requirements for the PC: either a multi-core processor, or hardware support for AVC decoding from the video card.

\subsection{Vector}

The advantage of vector formats over raster formats is the ability to scale to any screen resolution. This is useful for demonstrating the same file on different devices. Also vector graphic file size usually is small, it is important for transferring over the network and storing. Vector geoformats also bind elements to geocentric coordinates, as a result of which the image can be rasterized directly onto a sphere (geoid) without the need to use planar projections, as a result of which the shapes of objects, for example, near the poles, are not distorted.

Points, lines, polygons are usually used as geometric elements. Text and icons can also be added.

Animation mechanisms for vector formats can be combined of the following types:

- $\quad$ an array of sequentially rasterized vector files; 
- $\quad$ routes;

- style animation;

- vertex animation.

The simplest option is an array of sequentially rasterized vector files. At the same time, for smooth perception by the human eye, the animation flow rate should be at least 16 frames per second. As the duration of the animation increases, the number of files that need to be processed in a short time increases, which places demands on the computing components of a PC.

Animation by defining routes implements the movement of an icon along a trajectory determined by coordinates. It is usually implemented by simply assigning a specialized attribute to a polyline.

Style animation is set by changing some attribute over time, for example, fill color, movement of an object along a path, etc. Styled animation can be set outside the body of the geoformat description.

The most difficult option is animation of individual coordinates of curves, lines, polygons. In this case, vertex animation is required, which must be set internally by the vector format.

Among vector geoformats supported by HSC "Orbus", Shapefile was initially chosen as traditionally used by GIS users of GC RAS, as well as KML (KMZ) and GeoJSON (TopoJSON), which are output for REST API ArcGIS [19], and various web services: Openweather [20], USGS [21] etc. The following is a brief description of these formats.

\subsubsection{Shapefile}

Shapefile was developed by ESRI (the creator of ArcGIS) in the early 90s. This is the simplest geodata transfer format that allows storing two-dimensional or three-dimensional coordinates of geometric primitives, using various projections. Attribute information can be attached to each primitive [22].

A Shapefile is composed of multiple files in a binary format. Coordinates are stored in a separate file; all attribute information is stored in a single table also in a separate file. Due to the fact that the format is binary, the user cannot directly read and edit files.

Shapefile does not directly support topology, however it can be artificially injected [23].

From animation tools in this format, support for routes is implemented (you can set it as a polyline with a check mark in the attribute table).

Styles in Shapefile are not supported. However, there are separate implementations tied to specific programs, not interacting with each other.

\subsection{2. $\mathrm{KML}(\mathrm{KMZ})$}

KML (Keyhole Markup Language) is developed by Google. The last variant was adopted in 2015 [24].

KML includes some of the properties of the GML format (described below), but does not fully inherit this format, in addition, it describes the display styles of objects.

KML does not support splines and arcs. For lines, you can adjust color, transparency, thickness, for polygons - fill color and outline color.

$\mathrm{KMZ}$ is a compressed, archived version of KML.

\subsubsection{GeoJSON and TopoJSON}

GeoJSON (Geographic JavaScript Object Notation) was developed by the open standards organization IETF, the latest version of the standard was approved in 2016 [25]. GeoJSON is a JSON extension, it can be processed with a text editor.

In GeoJSON, standard two- and three-dimensional geometric primitives, as well as attribute information in the form of properties, are bound to each object.

Working with animation in the standard for GeoJSON is not described, as well as styling.

Users often apply CSS styles, one of the options is described in [26]. 
TopoJSON is an evolution of GeoJSON. It implements topology support when you define relationships between elements, and, for example, you can define a common border between areas. As a result, the size is reduced, but such a file is more difficult for a person to perceive; specialized libraries are needed to work with it in programs.

\section{ISV web platform graphic resources format selection and some features of their processing}

The use of the above raster and vector formats on the ISV web platform may be not always optimal. Modern browsers widely support a number of optimized raster and vector formats that can be easily adapted for web virtual globe.

\subsection{Raster}

WebP (Web Picture) is a widespread open-source image format proposed by Google in 2010. It uses lossy and lossless compression, animation and transparency. Lossless WebP images are 26\% smaller than PNG. WebP lossy images are 25-34\% smaller than comparable JPEG images at equivalent SSIM quality index. [27]

As of July 2021, 95.37\% of the browsers used worldwide are natively supported by WebP. [28]

Metadata can be added using EXIF and XMP technologies.

Among the limitations, one can single out that classically WebP does not work in the RGB color space: before encoding, the image is converted to YUV with a depth of 8 bits per channel and a 4:2:0 format according to the ITU-R BT.601 standard. To overcome these limitations, the WebP Lossless specification was developed, operating in the ARGB color space. [29].

Although it is also possible to use WebP to animate the surface of the planets, video textures are more convenient in presenting and transmitting over the network, moreover, WebGL engines have ways to process them natively [30]. Therefore, it was decided to store the raster image sequence in the media container. The choice fell on WebM, which supports VP8 video codec, which uses the equivalent WebP compression algorithms.

WebM (Web Media File) is an open media container format that is a subset of the Matroska media container (MKV, MKA). This format was presented by Google together with WebP at the Google I/O 2010 conference as a replacement for the proprietary MP4 container standards, and, accordingly, is suitable for use in non-commercial applications.

Designed as a container primarily providing VP8 video codec based on a still image (keyframe) compression algorithm.

As of July 2021, WebM/VP8 is natively supported by $96.57 \%$ of the browsers used worldwide [31]. However, MacOS (Safari browser) and iOS (most browsers) have limited support for WebM/VP8 due to Apple's promotion of proprietary graphic formats. In this case, it is appropriate to use the multiple sources technique [32], when the HTML5 <video> tag is given with several video sources (codecs), the browser program selects one of the sources, due to its support. The list of alternative web video codecs [33] should be reasonable: it is desirable that the codec is open-source, covering the maximum target platforms and supporting the alpha channel. The last feature is important for several semitransparent textures - video layers.

A comparative Table 1 of raster formats is shown below. 
Table 1

Raster formats

\begin{tabular}{cccccc}
\hline & Compression & $\begin{array}{c}\text { Depth per } \\
\text { channel }\end{array}$ & $\begin{array}{c}\text { Transpa- } \\
\text { rency }\end{array}$ & Animation & Metadata \\
\hline PNG & Lossless & Up to 16 bits & Yes & No & EXIF, XMP \\
JPEG & Lossy & 8 bits & No & No & EXIF, XMP \\
GeoTIFF & Adjustable & 32 bits/selectable & Yes & No (though layers) & Geotags \\
GIF & Lossless & Palette 256 colors & Binary & Yes & XMP \\
DDS & Lossy & Up to 32 bits & Yes & No & No \\
WebP & Lossless and lossy & 8 bits & Yes & Yes & EXIF, XMP \\
\hline
\end{tabular}

\subsection{Vector}

Among vector formats, GML geoformats were considered, the specification of which describes a wide range of possibilities, and SVG, which is not a geoformat, but allows a wide range of visual effects.

GML (Geography Markup Language) was developed by the Open Geospatial Consortium, an organization specializing in geospatial standards development. The latest version of the standard dates back to 2016 [34]. It is based on XML.

In addition to standard geometric primitives, GML supports arcs and curves defined by two and three coordinates. In addition, GML has the ability to work with topology.

GML can store raster data as well.

GML supports animation of vertices and various attributes. The change can be specified using intervals and specific time stamps. Styling is not described in the standard.

SVG (Scalable Vector Graphics) was developed by the organization responsible for the W3C technology standards. The last adopted standard dates back to 2011. The SVG2 version is currently being developed [35].

This format is based on XML, so it can be read and corrected with a text editor. In addition to the standard elements for all vector formats, arcs, Bézier curves (of the second and third order) are used. Bézier curves are a particularly powerful tool, with which the artist can draw any two-dimensional line using a small number of coordinates. All constructions in SVG are implemented on a flat canvas without 3D support and any projections.

SVG supports animation. It is possible to change the scale, color and other parameters in time, to transform the coordinates of the curves.

The standard also describes styling: applying colors, adjusting transparency, linear and radial gradients. In addition, it is possible to apply various visual effects to the image (blur, brightness, etc.). Styling, animation, and filtering can be done through CSS, which is widely supported at this time. However, some features, such as transforming curves and fine filtering settings are not supported in CSS.

The SVG format is also convenient for creating icons.

A comparative Table 2 of vector formats is shown below.

Table 2

Vector formats

\begin{tabular}{ccccccc}
\hline & $\begin{array}{c}\text { Splines } \\
\text { arcs }\end{array}$ & $\begin{array}{c}\text { Projec- } \\
\text { tions }\end{array}$ & $\begin{array}{c}\text { Attribute } \\
\text { informa- } \\
\text { tion }\end{array}$ & $\begin{array}{c}\text { Raster } \\
\text { data }\end{array}$ & Animation & Stylization \\
\hline SVG & Yes & No & No & Yes & Yes & Yes \\
Shapefile & No & Yes & Yes & No & No (except routes) & No \\
GeoJSON & No & Yes & Yes & No & No & No (realization with CSS) \\
GML & Yes & Yes & Yes & Yes & Yes & No \\
KML & No & Yes & Yes & Yes & Yes & Yes (no gradient, filters) \\
\hline
\end{tabular}




\section{Conclusions}

The main criteria for the raster format selection were high image quality, small file size, support for transparency and animation, native browser support, and open-source compliance. Raster format WebP satisfied and associated WebM media container all criteria (in the lossless specification).

However, in browser applications such as a virtual globe, when the user is interested in global processes, depth 8 bits per channel is acceptable in the vast majority of cases; otherwise, the combination of two WebP images can be considered.

Analysis of vector formats showed that GML has the broadest basic functionality among all the mentioned vector formats, the markup language of which is readable by any browser. At the same time, it does not provide the ability to set stylization. SVG format, which is natively supported by any browser, high-quality styling is implemented, but there is no 3D support and the ability to work with projections and coordinate systems, which makes it very difficult to rasterize a virtual globe in 3D space. In addition, attributive information about geo objects can be added to the SVG file using special tools, but software designed to work with this format usually does not provide such an option.

Therefore, SVG image creation with the capabilities of vector graphics editors with further conversion to a GML-like format supplemented by enriched styling such as CSS appears to be effective and can be the subject of the following research.

\section{Acknowledgements}

This work was conducted in the framework of budgetary funding of the Geophysical Center of RAS, adopted by the Ministry of Science and Higher Education of the Russian Federation.

This work employed facilities and data provided by the Shared Research Facility "Analytical Geomagnetic Data Center" of the Geophysical Center of RAS (http://ckp.gcras.ru/).

\section{References}

[1] S. Presnyakov, G. Boyarshinov, A. Odintsova, A. Rybkina, Spherical Visualization Database Prototype for Education and Scientific Research: Designing and Managing, in: Proceedings of the 30th International Conference on Computer Graphics and Machine Vision (GraphiCon 2020), Saint Petersburg, Russia, 2020, pp. 1-15. doi: 10.51130/graphicon-2020-2-3-12.

[2] A. Rybkina, A. Bobkov, O. Nikiforov, O. Pyatygina, Hardware and software system for visualization of geophysical data on a spherical screen, Scientific Visualization 7(2) (2015) 3849.

[3] Science Education through Earth Observation for High Schools (SEOS), URL: https://seosproject.eu/.

[4] UV mapping - Wikipedia, URL: https://en.wikipedia.org/wiki/UV_mapping.

[5] NASA Visible Earth - Home, URL: https://visibleearth.nasa.gov.

[6] NASA: Climate Change and Global Warming, URL: https://climate.nasa.gov.

[7] Home - NASA Solar System Exploration, URL: https://solarsystem.nasa.gov.

[8] Portable Network Graphics (PNG) Specification (Second Edition), URL: https://www.w3.org/TR/PNG/.

[9] ISO - ISO/IEC 10918-7:2019 - Information technology - Digital compression and coding of continuous-tone still images - Part 7: Reference software, URL: https://www.iso.org/standard/75845.html.

[10] Terminal equipment and protocols for telematic services, URL: https://www.w3.org/Graphics/JPEG/itu-t81.pdf.

[11] OGC GeoTIFF Standard, URL: http://docs.opengeospatial.org/is/19-008r4/19-008r4.html

[12] TIFF Revision 6, URL: https://www.adobe.io/content/dam/udp/en/open/standards/tiff/TIFF6.pdf.

[13] ISO - ISO 12639:2004 - Graphic technology - Prepress digital data exchange - Tag image file format for image technology (TIFF/IT), URL: https://www.iso.org/standard/34342.html. 
[14] Cover Sheet for the GIF89a Specification, URL: https://www.w3.org/Graphics/GIF/specgif89a.txt.

[15] DDS - Win32 apps | Microsoft Docs, URL: https://docs.microsoft.com/ru$\mathrm{ru} /$ windows/win32/direct3ddds/dx-graphics-dds.

[16] WEBGL_compressed_texture_s3tc - Web APIs | MDN, URL: https://developer.mozilla.org/enUS/docs/Web/API/WEBGL_compressed_texture_s3tc.

[17] ISO - ISO/IEC 14496-14:2003 - Information technology - Coding of audio-visual objects - Part 14: MP4 file format, URL: https://www.iso.org/standard/38538.html.

[18] MPEG LA's AVC License Will Not Charge Royalties for Internet Video that is Free to End Users through Life of License | Digital TV News, URL: https://www.digitaltvnews.net/?p=15979.

[19] Output formats-ArcGIS REST APIs | ArcGIS Developers, URL: https://developers.arcgis.com/rest/services-reference/enterprise/output-formats.htm.

[20] Current weather and forecast - OpenWeatherMap, URL: https://openweathermap.org.

[21] USGS Earthquake Hazards Program, URL: https://earthquake.usgs.gov.

[22] ESRI Shapefile Technical Description, URL: www.esri.com/content/dam/esrisites/sitecorearchive/Files/Pdfs/library/whitepapers/pdfs/shapefile.pdf.

[23] Understanding Topology and Shapefiles, URL: www.esri.com/news/arcuser/0401/topo.html.

[24] OGC 12-007r2, URL: https://docs.opengeospatial.org/is/12-007r2/12-007r2.html.

[25] rfc7946, URL: https://tools.ietf.org/html/rfc7946.

[26] Geojson CSS - OpenStreetMap Wiki, URL: https://wiki.openstreetmap.org/wiki/Geojson_CSS.

[27] A new image format for the Web | WebP | Google Developers, URL: https://developers.google.com/speed/webp/.

[28] WebP image format | Can I use... Support tables for HTML5, CSS3, etc, URL: https://caniuse.com/webp.

[29] WebP Lossless Bitstream Specification | Google Developers, URL: https://developers.google.com/speed/webp/docs/webp_lossless_bitstream_specification.

[30] Animating textures in WebGL - Web APIs | MDN, URL: https://developer.mozilla.org/enUS/docs/Web/API/WebGL_API/Tutorial/Animating_textures_in_WebGL.

[31] WebM image format | Can I use... Support tables for HTML5, CSS3, etc, URL: https://caniuse.com/webm.

[32] <video> $\quad-\quad$ HTML $\quad$ MDN, $\quad$ URL https://developer.mozilla.org/ru/docs/Web/HTML/Element/video\#multiple_sources_example.

[33] Web video codec guide - Web media technologies | MDN, URL: https://developer.mozilla.org/enUS/docs/Web/Media/Formats/Video_codecs.

[34] OpenGIS ${ }^{\circledR}$ Geography Markup Language (GML) Encoding Standard, URL: https://portal.ogc.org/files/?artifact_id=74183\&version=2.

[35] Scalable Vector Graphics (SVG) 2, URL: https://www.w3.org/TR/SVG2/. 